\title{
Low Incidence of Hemorrhagic Infarction Following Coronary Reperfusion with Nasaruplase in a Canine Model of Acute Myocardial Infarction
}

\author{
Comparison with Recombinant t-PA
}

\author{
Hideaki Kido, Ph.D., Kazutaka Hayashi, M.S., \\ Takeshi UahIDA, Ph.D. \\ and Masahiro Watanabe, Ph.D.
}

\section{Summary}

An examination was made of the coronary thrombolytic effects of nasaruplase in a canine model of acute myocardial infarction. The model was produced by selective injection of an artificial thrombus into the coronary artery stenosed by laser ablation. Intravenous nasaruplase $(8 \mathrm{U} / \mathrm{kg} / \mathrm{min})$ showed an equivalent thrombolytic effect to a recombinant tissue-type plasminogen activator (rt-PA, $10,000 \mathrm{IU} / \mathrm{kg} / \mathrm{min}$ ) as assessed by reperfusion rate $(78.6$ versus $79.2 \%$ ) and reperfusion time (37.4 \pm 5.2 versus $37.0 \pm 2.5 \mathrm{~min})$. Nasaruplase decreased the plasma $\alpha_{2}$-plasmin inhibitor $\left(\alpha_{2}\right.$-PI level by $28 \%$ immediately after reperfusion, but hardly altered fibrinogen or plasmin- $\alpha_{2}$-plasmin inhibitor complex (PIC) levels. By contrast, rt-PA significantly decreased plasma $\alpha_{2}$-PI and fibrinogen levels, by $84 \%$ and $92 \%$ respectively, and, increased PIC level more than 70-fold. Hemorrhagic infarction occurred in 2 of 14 animals in the nasaruplase group and in 9 of 19 animals in the rt-PA group. In these animals, significant correlations were found between the ratio of the hemorrhagic infarction area to total infarct area and the plasma $\alpha_{2}$ PI $(r=-0.740, p<0.05)$ or fibrinogen $(r=-0.798, p<0.05)$ concentrations, as well as between the recovery rate of left ventricular regional wall motion and the plasma $\alpha_{2}$-PI $\langle r=0.924, p<0.01)$ or fibrinogen $(r=0.864, p<0.01)$ concentrations. It is concluded that nasaruplase is a potent thrombolytic agent which preserves left ventricular function with a lesser rate of hemorrhagic infarction than rt-PA. Further, nasaruplase administration results in recovery of left ventricular regional wall motion and systolic function, such as Vmax. (Jpn Heart J 35: 6179, 1995)

Key words: nasaruplase scu-PA myocardial infarction animal model thrombolysis systemic fibrinogenolysis hemorrhagic infarction left ventricular function t-PA reocclusion

From the Department of Pharmacology, Product Development Laboratories, The Green Cross Corporation, Osaka, Japan.

Address for correspondence: Hideaki Kido, Ph.D., Department of Pharmacology, Product Development

Laboratories, The Green Cross Corporation, 2-25-1, Shodai-Ohtani, Hirakata, Osaka 573, Japan.

Received for publication July 8, 1994.

Accepted September 20, 1994. 
7 ISSUE-TYPE plasminogen activator (t-PA) is widely used for thrombolytic 1 therapy in acute myocardial infarction (AMI). ${ }^{1)}$ Although t-PA shortens thrombolysis time and raises the reperfusion rate compared with either urokinase (UK) or streptokinase $(\mathrm{SK}),{ }^{2-4)}$ bleeding complications remain a risk of t-PA therapy. ${ }^{5,6)}$ Although hemorrhagic infarction has generally been considered a reperfusion injury, ${ }^{7)}$ the incidence of hemorrhagic infarction has been found to be increased by thrombolytic agents, ${ }^{8}{ }^{8}$ occurring in about half of patients receiving such agents. ${ }^{9)}$ Hemorrhagic infarction is observed within the reperfused area of the ischemic myocardium. ${ }^{10)}$ However, there exists the opposite opinion that hemorrhagic infarction may enlarge the infarct size beyond the reperfused area. ${ }^{11)}$ In fact, the mass of hemorrhagic infarction varies and the factors determining hemorrhagic infarct size remain obscure.

Nasaruplase is a native single-chain UK-type plasminogen activator (scuPA) derived from a human kidney cell line. It is a proenzyme converted only at the thrombus surface. ${ }^{12-14)}$ Nasaruplase has higher selectivity than UK for fibrin lysis. ${ }^{13,15)}$ Intracoronary administration of nasaruplase in humans has been shown to recanalize the occluded coronary arteries in $90 \%$ of patients while showing weaker systemic fibrinogenolytic effects than UK. ${ }^{16)}$ In the present study, we compared the thrombolytic effects and incidence of hemorrhagic infarction and its relation to left ventricular function and infarct size after intravenous administration of nasaruplase to t-PA in a canine model of AMI.

\section{Materials and Methods}

Animals: Adult mongrel dogs of both sexes (KEARI, Japan) weighing 8 to $19 \mathrm{~kg}$ were anesthetised by intravenous injection of $30 \mathrm{mg} / \mathrm{kg}$ of sodium pentobarbital, intubated and ventilated with room air at a rate of 20 respirations $/ \mathrm{min}$ with a $20 \mathrm{~m} l / \mathrm{kg}$ stroke volume.

Thrombolytic agents: Nasaruplase $(400 \mathrm{U} / \mathrm{mg})$ was highly purified from the culture medium of a human kidney cell line (Green Cross Corp., Japan). t-PA was a recombinant product (Activasin ${ }^{\oplus}, 600,000 \mathrm{IU} / \mathrm{m}$ l, Kyowa Hakko Kogyo Co., Japan). Each agent, dissolved in physiological saline, was prepared immediately before use.

Animal model of acute myocardial infarction: A guiding catheter $(9 \mathrm{~F}$, Medtronic) was inserted into the ostium of the left coronary artery through the right carotid artery of the dog under fluoroscopic guidance. After coronary artery angiography (CAG) and left ventriculography (LVG) to define the baseline state, a $1.7 \mathrm{~mm}$ hot-tip YAG laser probe (MYL-3, Olympus, Japan) was inserted into the middle segment of the left anterior descending coronary artery $(\mathrm{LAD})$ and the artery ablated at $14 \mathrm{~W}$ for $2 \mathrm{sec}$ to produce a 50 to $90 \%$ stenosis. 
The artificial thrombus was made by mixing arterial blood $(2 \mathrm{~m} l)$, fibrinogen (20 mg, Green Cross Corp.) and thrombin (30 units, Green Cross Corp.). The thrombus $(2 \times 2 \times 8 \mathrm{~mm}$ in size $)$ was then injected into the stenosed portion of the LAD to initiate AMI. Thirty minutes later, nasaruplase $(8 \mathrm{U} / \mathrm{kg} /$ $\mathrm{min}$ ) or $\mathrm{rt}-\mathrm{PA}(10,000 \mathrm{IU} / \mathrm{kg} / \mathrm{min})$, each dose corresponding to about 1.5 times that used in the respective clinical studies, was administered intravenously after a bolus injection of $2500 \mathrm{IU}$ heparin. Reperfusion was defined as successful when recanalization was verified by CAG or when a reperfusion arrhythmia occurred within $60 \mathrm{~min}$. When reperfusion was attained, the thrombolytic agent was stopped. Animals which developed ventricular fibrillation due to reperfusion arrhythmias were defibrillated. Defibrillation induced bradycardia in some animals, but the heart rate recovered rapidly. The catheters were then removed and the surgical incision in the neck was sutured. Antibiotics (penicillin and kanamycin) were injected intramuscularly. All dogs were cared for according to institutional guidelines.

The dogs were again anesthetized 1 week later, and a $9 \mathrm{~F}$ guiding catheter was inserted into the left carotid artery. Coronary reocclusion was assessed by GAG, and LVG was performed to measure left ventricular function. Thrombolytic therapy was not performed in the control group.

Determination of plasma concentrations of fibrinogen, $\alpha_{2}$-plasmin inhibitor $\left(\alpha_{2}-\mathrm{PI}\right)$, plasmin- $\alpha_{2}$-plasmin inhibitor complex (PIC) and thrombin antithrombin III complex (TAT): Blood samples were taken from the carotid artery prior to coronary artery occlusion, at $30 \mathrm{~min}$ after occlusion, immediately after reperfusion and after a week recovery period. Plasma was obtained immediately from $9 \mathrm{~m} l$ of whole blood to which $1 \mathrm{~m} l$ of $3.2 \%$ trisodium citrate was added. All plasma samples were kept frozen for later analysis. Commercially available kits were used for the measurement of fibrinogen (International Reagent Co., Japan), $\alpha_{2}$-PI (Daiichi Chemical Co., Japan), PIC (Teijin, Japan) and TAT (Teijin).

Electrocardiogram and hemodynamic measurements: Lead II electrocardiograms (ECG) were monitored throughout the experiment. Systemic arterial blood pressure (BP) was measured with a pressure transducer (Statham P-50, Gould). The heart rate (HR) was analyzed with a tachometer (Nihon Kohden, Japan). A catheter-tip pressure transducer (MPC-500, Millar Instruments) was inserted into the left ventricle through the right femoral artery to measure left ventricular end-diastolic pressure (LVEDP), $\mathrm{LV} \mathrm{dP} / \mathrm{dt}, \mathrm{LV} \mathrm{dP} / \mathrm{dt} / \mathrm{P}, \mathrm{V}_{\max }$ and time constant $(\mathrm{T})$. Cardiac output $(\mathrm{CO})$, pulmonary arterial blood pressure (PAP) and pulmonary capillary wedge pressure (PCWP) were measured using a $5 \mathrm{~F}$ Swan-Ganz catheter (TC-504, Viggo-Spectramed). Stroke volume (SV), systemic vascular resistance (SVR), total pulmonary vascular resistance (TPR) and pulmo- 
nary vascular resistance (PVR) were calculated from standard formulae. Parameters were recorded continuously with a polygraph system (RM-6000, Nihon Kohden).

Measurements of left ventricular function: The left ventricular ejection fraction (LVEF) and regional wall motion (rWM) were calculated from $30^{\circ}$ right anterior oblique ventriculograms with a computer analysis system (CAD-98, Nishimoto Industry, Japan) using Dodge's method ${ }^{17)}$ and the radial-area method, ${ }^{18)}$ respectively.

Quantification of infarct size: The animals were sacrificed with a lethal dose of pentobarbital sodium one week after infarction. The hearts were excised, weighed, cut cross-sectionally into plates $8 \mathrm{~mm}$ thick and stained with $0.5 \%$ 2,3,5-triphenyltetrazolium chloride solution (TTC). The infarct size was identified as the non-TTC-stained area. Relative cardiac (heart/body) weight was also calculated as an indicator of cardiac hypertrophy induced by myocardial infarction. Hemorrhagic infarction was quantified gross anatomically and histologically, and the hemorrhagic infarction ratio was calculated as:

$$
\text { hemorrhagic infarction ratio }(\%)=\frac{\text { area of hemorrhagic infarction }}{\text { total infarct area }} \times 100
$$

Statistical analysis: Results are expressed as mean \pm SEM. The frequency of hemorrhagic infarction or reocclusion was statistically analysed with a $\chi^{2}$ test. The survival rate was analysed using the Kaplan-Meier method. Values between two groups were analysed using the two-tailed nonpaired $t$ test. Other parameters were analysed applying Tukey's method for multiple comparisons. The results were considered significantly different if $p<0.05$.

\section{Results}

Thrombolytic effect: Representative photographs of the results of thrombolytic therapy are shown in Figure 1. During $30 \mathrm{~min}$ following coronary artery occlusion, the thrombus expanded on the upstream side of the LAD (Figure 1-C). When nasaruplase was administered intravenously after $30 \mathrm{~min}$ of coronary artery occlusion, coronary recanalization was achieved in $78.6 \%$ (11) 14) of animals within 20 to $55 \mathrm{~min}$ after the start of infusion (Figure l-D). rt-PA induced successful thrombolysis in $79.2 \%(19 / 24)$ of animals within 20 to $50 \mathrm{~min}$ after the start of infusion. The average time to reperfusion was $37.4 \pm 5.2 \mathrm{~min}$ in the nasaruplase group and $37.0 \pm 2.5 \mathrm{~min}$ in the rt-PA group (Table I). Spontaneous recanalization occurred in none of the control animals during a 1 week observation period. 


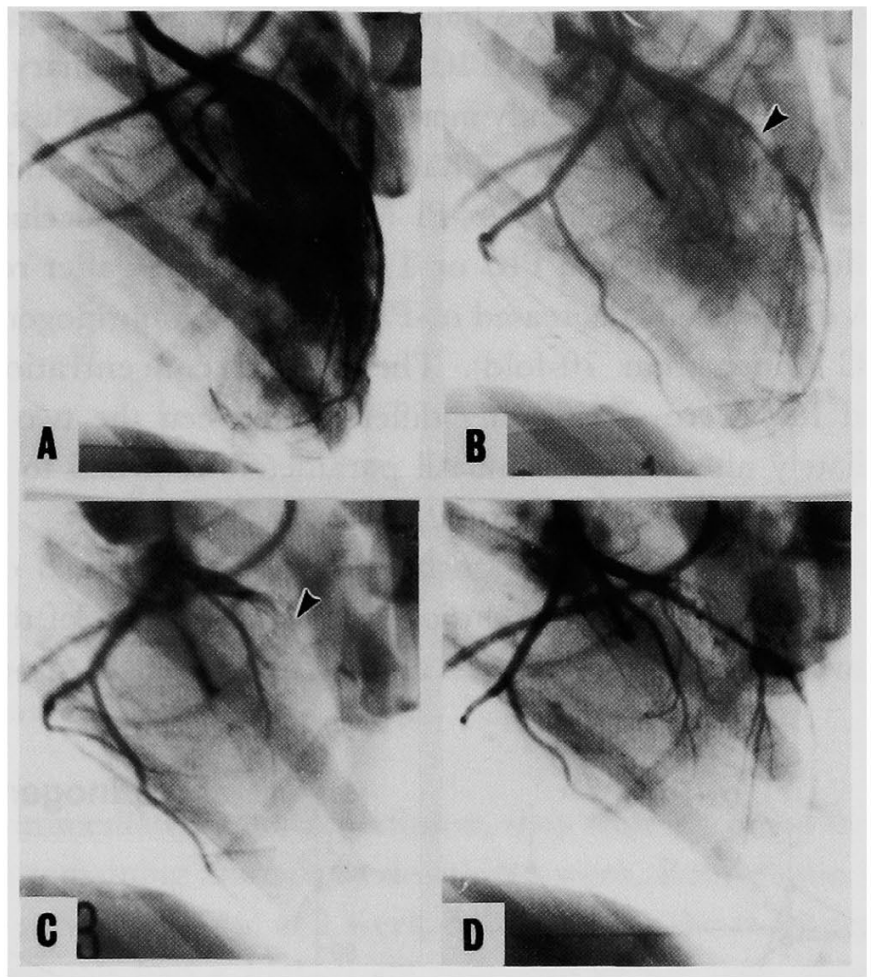

Figure 1. Coronary artery angiograms from a canine model of acute myocardial infarction (AMI). A: Before coronary artery occlusion (standard state), B: Stenosis of the coronary artery induced by laser ablation, $\mathrm{C}$ : After injection of an artificial thrombus, D: Immediately after reperfusion

Table I. Effects of Nasaruplase and rt-PA on Thrombolysis and Reocclusion

\begin{tabular}{ccc}
\hline & Nasaruplase & rt-PA \\
\hline Thrombolytic effect & & \\
Reperfusion rate & $11 / 14(78.6 \%)$ & $19 / 24(79.2 \%)$ \\
Reperfusion time & $37.4 \pm 5.2 \mathrm{~min}$ & $37.0 \pm 2.5 \mathrm{~min}$ \\
Perfusion insufficiency l week later & $1 / 8(12.5 \%)$ & $3 / 10(30.0 \%)$ \\
$\quad$ Reocclusion & 0 & $1 / 10(10.0 \%)$ \\
Restenosis & 0 & $1 / 10(10.0 \%)$ \\
No flow at apex & 0 & \\
\hline
\end{tabular}

In the nasaruplase group, coronary recanalization was achieved in 11 of 14 animals. Three of the 11 animals died within 1 week. Coronary reocclusion was observed in 1 of the 8 survivors at 1 week. In the rt-PA group, coronary recanalization was achieved in 19 of 24 animals. Since 5 of the 19 animals developed cardiac arrest immediately after reperfusion, they were excluded from the study. Four of the 14 remaining animals died within 1 week. Reocclusion was observed in 3 of the 10 survivors at 1 week. Moreover, 1 of the 7 non-reoccluded animals had a high-grade restenosis at the laser ablation site and one animal had circulatory insufficiency at the apex in the rt-PA group. 
Changes in plasma concentrations of $\alpha_{2}$-PI, fibrinogen, PIC and TAT: There were no significant differences between the three groups regarding plasma concentrations of $\alpha_{2}$-PI, fibrinogen, PIC or TAT before coronary artery occlusion (Table II). TAT was significantly increased $30 \mathrm{~min}$ after occlusion, but $\alpha_{2}$-PI, fibrinogen and PIC showed little change at this stage. Administration of nasaruplase significantly decreased $\alpha_{2}$ PI $(-28 \%$ vs before occlusion) but displayed little effect on fibrinogen, PIC or TAT immediately after reperfusion. In contrast, rt-PA dramatically decreased $\alpha_{2}$-PI $(-84 \%)$ and fibrinogen $(-92 \%)$ and increased PIC (more than 70-fold). The plasma concentrations of $\alpha_{2}$-PI, fibrinogen and PIC were significantly different between the two thrombolytic agents immediately after reperfusion. All parameters returned to pre-occlusion levels at 1 week post infarction (Figure 2).

Survival and reocclusion rates: In the nasaruplase group, 3 of 11 animals died within 1 week. Coronary reocclusion was observed in 1 of the 8 survivors $(12.5 \%)$ at 1 week. Since 5 of the 19 animals in the rt-PA group developed
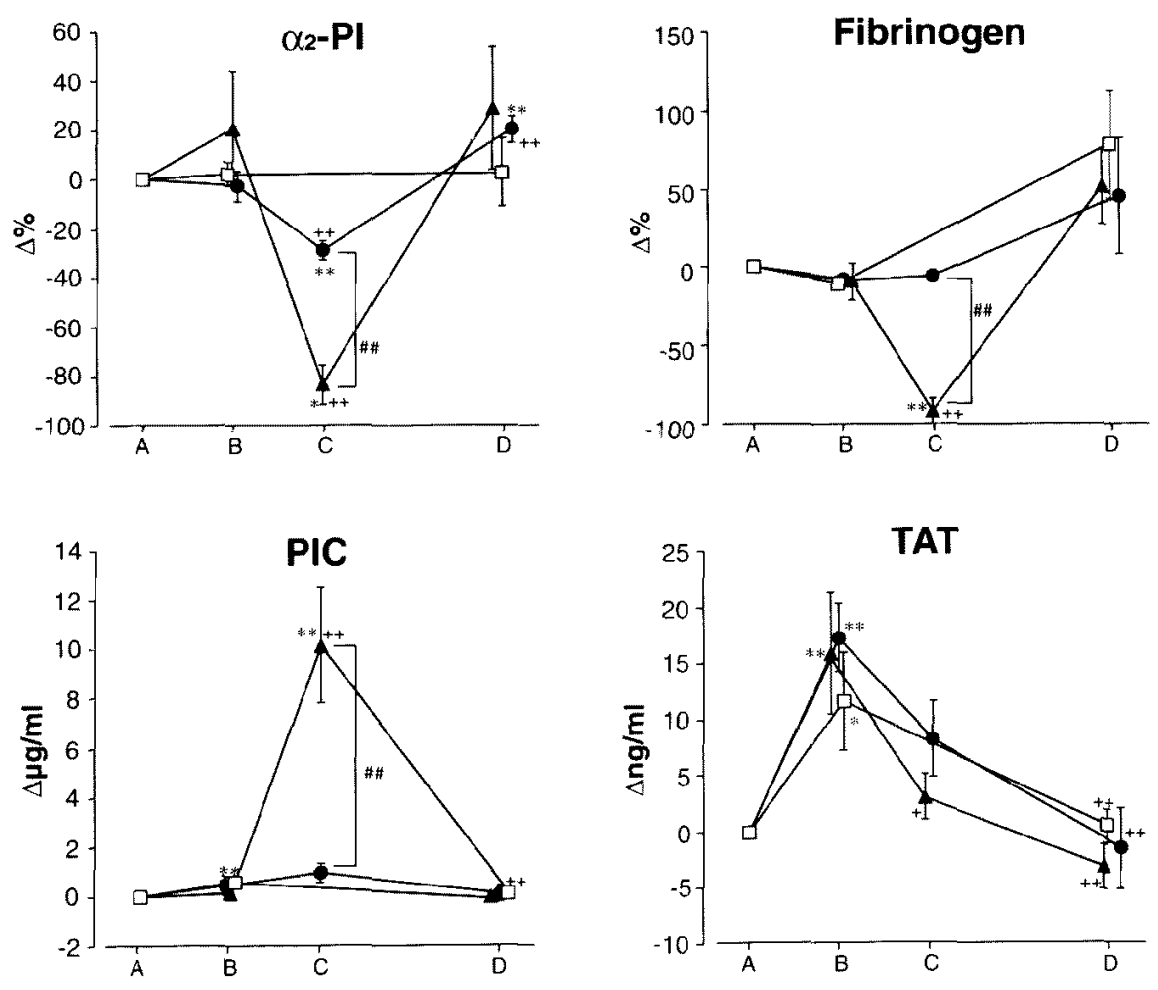

Figure 2. Changes in plasma concentrations of $\alpha_{2}$ PI, fibrinogen, PIC and TAT. $\boldsymbol{O}=$ nasaruplase $(n=7) ; \boldsymbol{\Delta}=\mathrm{rt}-\mathrm{PA}(n=7) ; \square=$ control $(n=7)$. A: Before coronary artery occlusion, B: $30 \mathrm{~min}$ after thrombus injection, $\mathrm{C}$ : Immediately after reperfusion, D: 1 week later; vertical bars represent SEM $*: p<0.05, * *: p<0.01$ vs $\mathrm{A},+$ : $p<0.05,+: p<0.01$ vs $\mathrm{B}, \# \#: p<0.01$ 


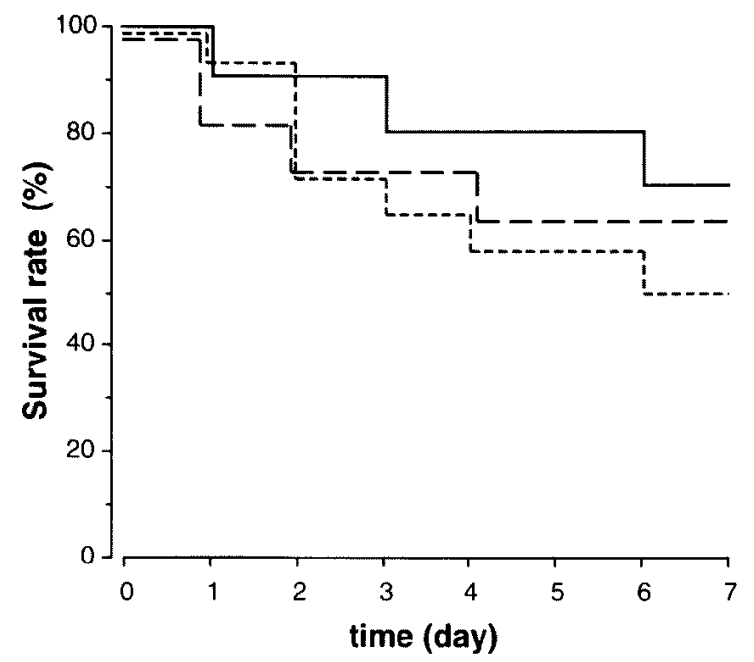

Figure 3. Time course of survival rates in a canine model of AMI. nasaruplase $(n=10), \cdots$, rt-PA $(n=11), \cdots$ : control $(n=14)$ Reoccluded animals were excluded from the analysis.

cardiac arrest immediately after reperfusion, they were excluded from the study. Four of the 14 remaining animals died within 1 week. Reocclusion was observed in 3 of the 10 survivors $(30 \%)$ at 1 week. Moreover, in the rt-PA group, 1 of the 7 remaining animals had a high-grade restenosis at the laser ablation site and another animal had circulatory insufficiency at the apex (Table I). Seven of 14 animals died within 1 week in the control group. The survival rates in the nasaruplase, rt-PA and control group, excluding reoccluded animals, were $70 \%$ (7/10), 62.5\% (7/11) and 50\% (7/14), respectively (Figure 3).

Electrocardiogram: ST-segment and T-wave elevations were observed immediately after coronary artery occlusion in this canine model of AMI. Treatment with nasaruplase and rt-PA reduced these elevations immediately after reperfusion. Reperfusion arrhythmias were observed infrequently for the next 5 to $10 \mathrm{~min}$. Premature ventricular contractions, atrioventricular block, ventricular tachycardia and ventricular fibrillation then gradually appeared. After 1 week, the $\mathrm{T}$-wave was elevated slightly in a few animals from both thrombolytic agent groups. Abnormal Q-waves were observed in 2 of the 7 animals of the control group.

Hemodynamics: There were no statistically significant differences in hemodynamics between the three groups before coronary artery occlusion (Table II). There was no significant change in $\mathrm{BP}$ or $\mathrm{HR}$. The $\mathrm{CO}$ and $\mathrm{SV}$ decreased gradually in the control group as a result of sustained coronary occlusion. No early recovery of $\mathrm{CO}$ and $\mathrm{SV}$ immediately after reperfusion with either nasaruplase or rt-PA was observed; however, $\mathrm{CO}$ and SV returned to pre-occlu- 
Table II. Baseline Hemostatic and Hemodynamic Characteristics

\begin{tabular}{|c|c|c|c|c|}
\hline & nasaruplase & rtwPA & control & $p$ value \\
\hline \multicolumn{5}{|l|}{ Hemostatic characteristics } \\
\hline$\alpha_{2}-\mu I(\%$ vs standard plasma) & $86.1 \pm 7.2$ & $90.0 \pm 19.2$ & $81.3 \pm 16.0$ & NS \\
\hline fibrinogen $(\mathrm{mg} / \mathrm{m} l)$ & $2.24 \pm 0.34$ & $2.44 \pm 0.31$ & $1.85 \pm 0.10$ & NS \\
\hline PIC $(\mu \mathrm{g} / \mathrm{m} l)$ & $0.20 \pm 0.11$ & $0.14 \pm 0.10$ & $0.07 \pm 0.05$ & NS \\
\hline $\mathrm{TAT}(\mathrm{ng} / \mathrm{m} l)$ & $7.28 \pm 2.04$ & $6.68 \pm 2.09$ & $4.63 \pm 1.01$ & NS \\
\hline \multicolumn{5}{|l|}{ Hemodynamic characteristics } \\
\hline \multicolumn{5}{|l|}{ 1) Systemic circulation } \\
\hline mean $\mathrm{BP}(\mathrm{mmHg})$ & $129 \pm 7$ & $130 \pm 7$ & $136 \pm 8$ & NS \\
\hline HR (beats/min) & $152 \pm 9$ & $143 \pm 10$ & $155 \pm 10$ & NS \\
\hline $\mathrm{CO}(l / \mathrm{min})$ & $2.2 \pm 0.2$ & $2.2 \pm 0.3$ & $1.9 \pm 0.3$ & NS \\
\hline $\mathrm{SV}(\mathrm{m} / /$ beat $)$ & $14.9 \pm 2.1$ & $15.3 \pm 1.2$ & $12.0 \pm 1.2$ & NS \\
\hline SVR (dynes.sec/ $\mathrm{cm}^{5}$ ) & $4457 \pm 429$ & $5029 \pm 547$ & $6312 \pm 697$ & NS \\
\hline LVEDP (mmHg) & $4.1 \pm 1.0$ & $3.7 \pm 0.6$ & $5.4 \pm 0.8$ & NS \\
\hline \multicolumn{5}{|l|}{ 2) Pulmonary circulation } \\
\hline mean PAP (mmHg) & $16 \pm 3$ & $20 \pm 2$ & $20 \pm 2$ & NS \\
\hline PCWP $(\mathrm{mmHg})$ & $8 \pm 2$ & $11 \pm 3$ & $11 \pm 2$ & NS \\
\hline TPR (dynes.sec/ $/ \mathrm{cm}^{5}$ ) & $574 \pm 73$ & $851 \pm 202$ & $953 \pm 162$ & NS \\
\hline PVR (dynes.sec/ $/ \mathrm{cm}^{5}$ ) & $201 \pm 31$ & $296 \pm 56$ & $418 \pm 93$ & NS \\
\hline \multicolumn{5}{|l|}{ 3) Left ventricular function } \\
\hline$+\mathrm{dP} / \mathrm{dt} \max (\mathrm{mmHg} / \mathrm{sec})$ & $3010 \pm 330$ & $2880 \pm 400$ & $3450 \pm 350$ & NS \\
\hline$+\mathrm{dP} / \mathrm{dt} / \mathrm{P} \max (/ \mathrm{sec}\rangle$ & $34.7 \pm 4.6$ & $56.5 \pm 5.7$ & $61.5 \pm 3.5$ & NS \\
\hline$V \max (/ \mathrm{sec})$ & $79.2 \pm 7.4$ & $74.8 \pm 6.6$ & $81.0 \pm 3.1$ & NS \\
\hline$-\mathrm{dP} / \mathrm{dt} \min (\mathrm{mmHg} / \mathrm{sec})$ & $-3680 \pm 180$ & $-3110 \pm 210$ & $-4350 \pm 520$ & NS \\
\hline $\mathrm{T}$ (msec) & $26.5 \pm 1.6$ & $32.1 \pm 1.9$ & $25.8 \pm 3.3$ & NS \\
\hline
\end{tabular}

NS: not significant

sion levels after 1 week, especially in the nasaruplase group. The SVR and LVEDP rose during AMI and recovered 1 week after thrombolytic therapy. There was a significant difference in LVEDP between the nasaruplase group and the control group at 1 week. The mean PAP, PCWP, TPR and PVR gradually rose in the control group for 1 week. These parameters recovered to baseline at 1 week in each thrombolytic agent-treated group.

\section{Left ventricular function:}

Left ventriculographic investigation: The LVEF was lowered significantly by AMI and did not recover in the control group 1 week later. Left ventricular function in the nasaruplase group was improved 1 week after reperfusion, but not immediately after reperfusion. The effect of rt-PA was weaker than that of nasaruplase (Figure 4).

Regional wall motion (rWM) in segments 1,2 and 3, corresponding to the left ventricular anterobasal, anterolateral and apical portions, respectively, was reduced significantly by AMI, compared to baseline. The rWM in segment 3 improved after 1 week, but segment 2 was unchanged in the control group. A significant improvement of rWM in segments 1 and 2 was observed in the nasaruplase group 1 week after reperfusion. There was only slight improvement 


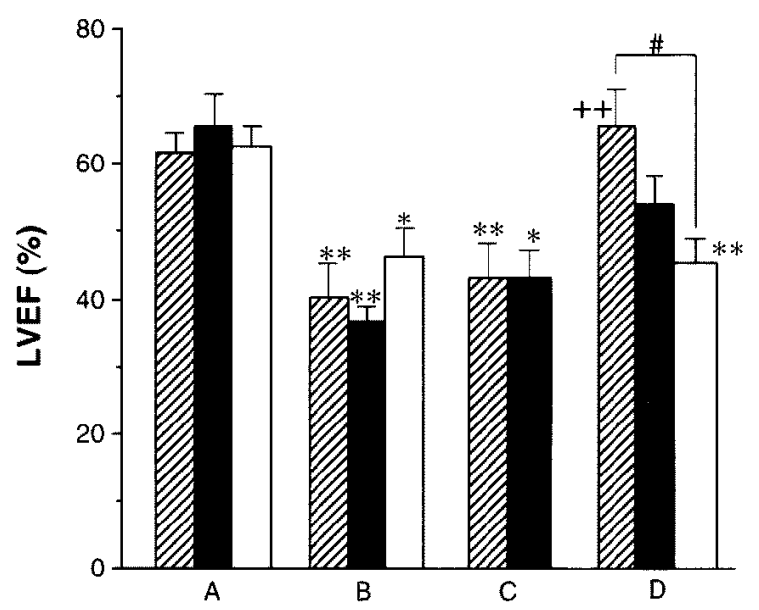

Figure 4. Changes in left ventricular ejection fraction. $\square=$ nasaruplase $(n=7)$, $\mathbf{\square}=\mathrm{rt}-\mathrm{PA}(n=7), \square=\mathrm{control}(n=7)$. A: Before coronary artery occlusion, B: $30 \mathrm{~min}$ after thrombus injection, $C$ : Immediately after reperfusion, $D: 1$ week later. Vertical bars represent SEM ${ }^{*}: p<0.05, * *: p<0.01$ vs $\mathrm{A},+: p<0.01$ vs $\mathrm{B}, \#: p<0.05$

of rWM in the rt-PA group (Figure 5). In segments 4 (diaphragmatic) and 5 (posterobasal) corresponding to non-infarct area, rWM also showed the tendency to decrease following the occlusion of LAD, but the degree was slight and not significant.

Hemodynamic investigation: $\mathrm{V}_{\max }$, which indicates left ventricular systolic function, decreased following AMI. A further fall was observed at 1 week in the control group. Nasaruplase slightly improved these parameters immediately after reperfusion and they improved significantly at 1 week post infarction compared to $30 \mathrm{~min}$ after coronary occlusion and to the parameters in the control group. The improvement of LV function after rt-PA, compared with that produced by nasaruplase, was slight (Figure 6).

A T prolongation was observed after AMI, which indicates deterioration of left ventricular diastolic function. Nasaruplase and rt-PA did not improve LV diastolic function immediately after reperfusion. There were no differences in this parameter between the three groups at 1 week (Figure 6).

Anatomic findings: In the control group widespread transmural necrosis was commonly observed at 1 week, between the left ventricular anterolateral portion and the apex, representing the area perfused by the occluded vessel. The necrotic myocardium was denatured and fibrotic. Necrosis in the nasaruplase and rt-PA groups was generally localized to the internal area of the left ventricle. Hemorrhagic infarction was observed in 2 of the 14 animals in the nasaruplase and control group (including dead animals), at up to 1 week. By contrast, the frequency of hemorrhagic infarction in the rt-PA group (9/19) was significantly 

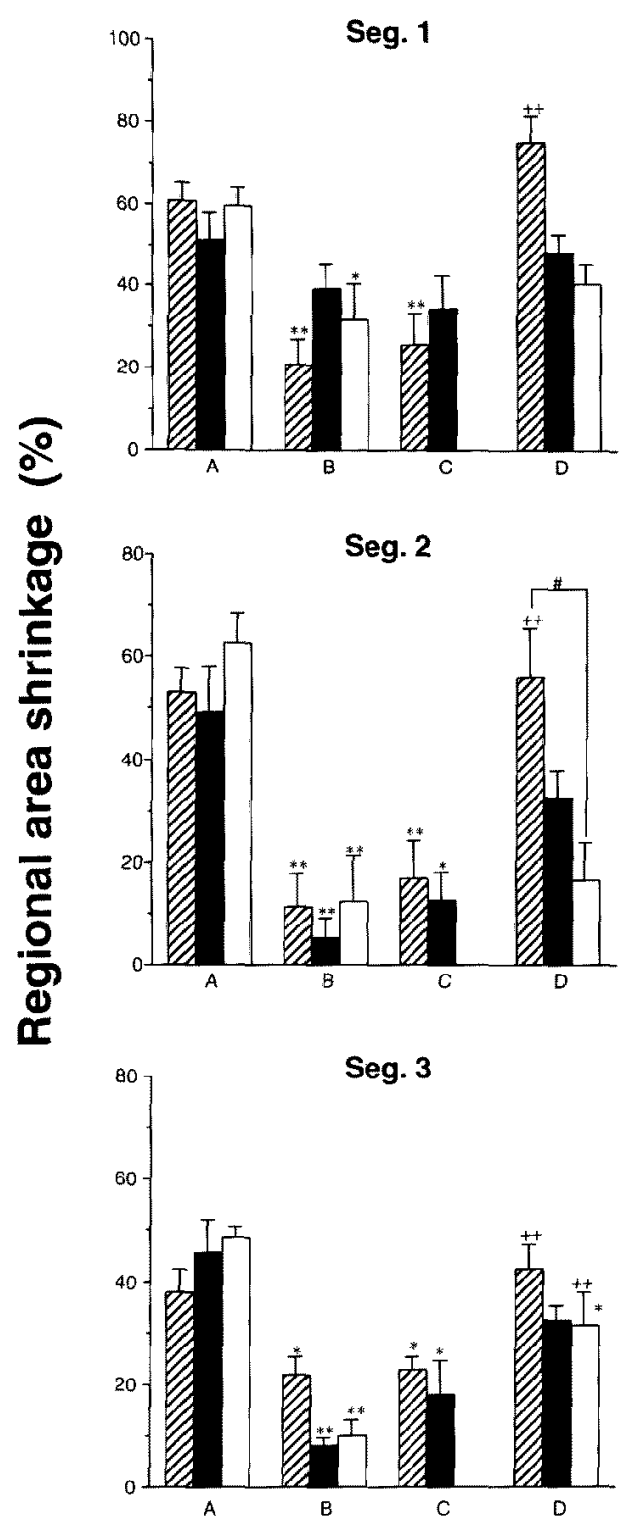

Figure 5. Changes in left ventricular regional wall motion assessed by regional area shrinkage. Seg. 1: Anterobasal, Seg. 2: Anterolateral, Seg. 3: Apical portion. $\square=$ nasaruplase $(n=7) ; \boldsymbol{\square}=\mathrm{rt}-\mathrm{PA}(n=7) ; \square=$ control $(n=7)$ A: Before coronary artery occlusion, B: $30 \mathrm{~min}$ after thrombus injection, C: Immediately after reperfusion, D: 1 week later. Vertical bars represent SEM. *:p<0.05,**:p<0.01 vs A, +*: $p<0.01$ vs $\mathrm{B}, \#: p<0.05$

higher than in the other two groups (Figure 7, Table III).

Infarct size and relative cardiac weight: Infarct sizes were $4.6 \pm 2.7,5.2 \pm$ 2.5 and $25.7 \pm 6.2 \%$ in the nasaruplase, rt-PA and control groups, respectively. 

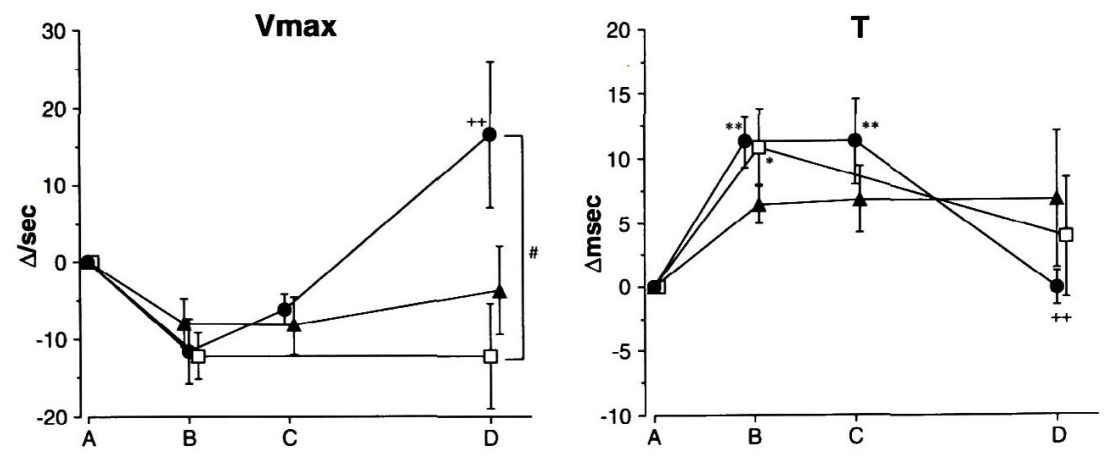

Figure 6. Changes in left ventricular systolic (Vmax) and diastolic (T) function. $=$ nasaruplase $(n=7) ; \boldsymbol{\Delta}=$ rt-PA $(n=7) ; \square=$ control $(n=7)$. A: Before coronary artery occlusion, B: $30 \mathrm{~min}$ after thrombus injection, C: Immediately after reperfusion, D: 1 week later. Vertical bars represent SEM. $*: p<0.05, * *: p<0.01$ vs A, H: $p<0.01$ vs $\mathrm{B}, \#: p<0.05$

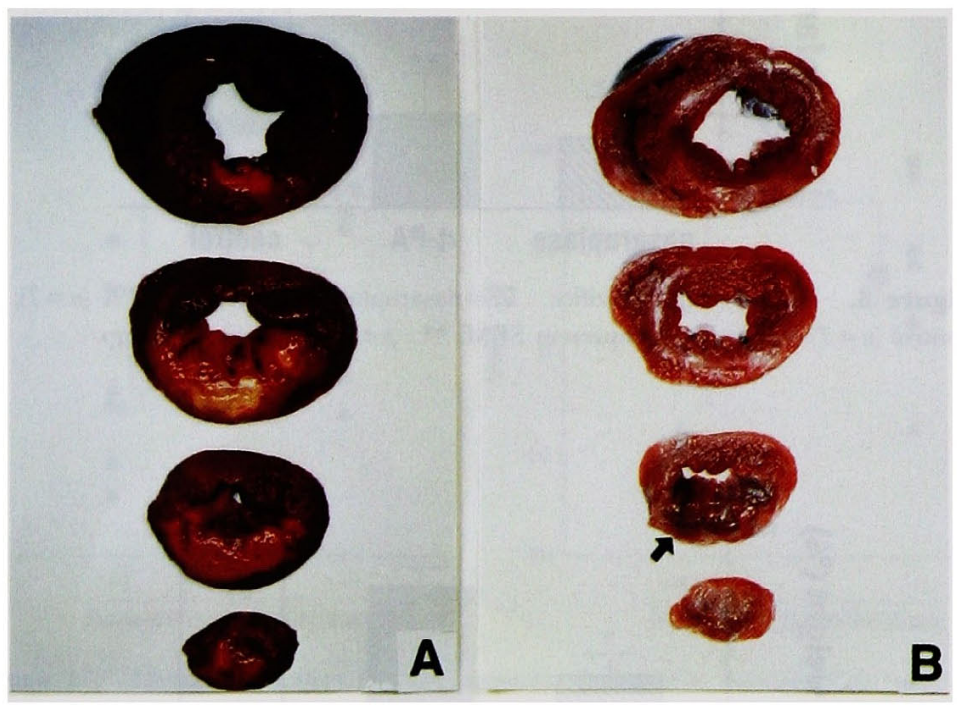

Figure 7. Infarct area at sacrifice. A: Fibrosis in a control dog; myocardium stained with 2,3,5-triphenyltetrazolium chloride (TTC). B: Hemorrhagic infarction in an rtPA-treated dog (arrow).

Nasaruplase and rt-PA reduced infarct size to an equal degree (Figure 8). The relative cardiac weights were $0.70 \pm 0.04,0.85 \pm 0.05$ and $0.97 \pm 0.09 \%$ in the nasaruplase, rt-PA and control groups, respectively. Nasaruplase significantly suppressed cardiac hypertrophy subsequent to AMI as assessed by relative cardiac weight. The effect in the rt-PA group was not significantly different from the control group (Figure 9).

Correlation between hemorrhagic infarction and other parameters: A sig- 
Table III. Frequency of Hemorrhagic Infarction by Intravenous Administration of Thrombolytic Agents in a Canine Model of Acute Myocardial Infarction

\begin{tabular}{llcc}
\hline \multirow{2}{*}{ Group } & \multicolumn{2}{l}{ Hemorrhagic infarction } & Significance \\
\cline { 2 - 3 } & $(+)$ & $(-)$ & \\
\hline Control & 2 & 12 & \\
Nasaruplase & 2 & 12 & $t<0.05$ \\
rt-PA & 9 & 10 & \\
\hline
\end{tabular}

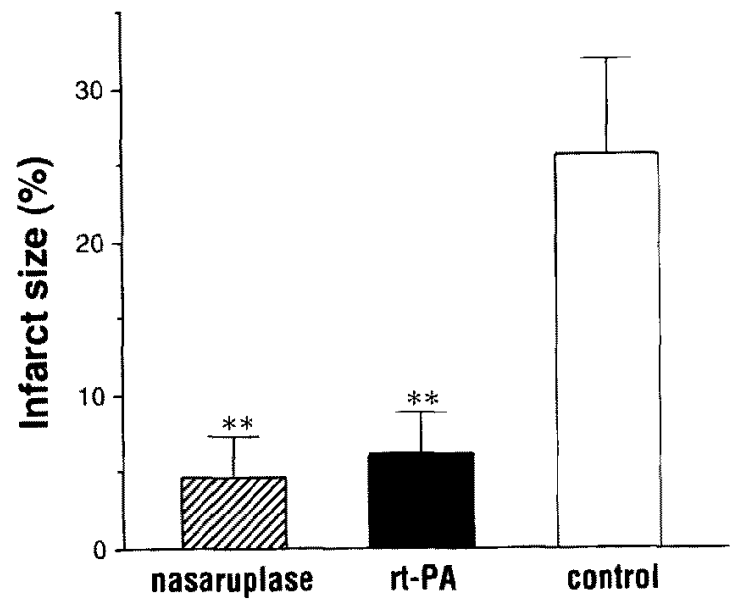

Figure 8. Infarct size at sacrifice. $Z=$ nasaruplase $(n=7), \square=$ rt-PA $(n=7)$, control $(n=7)$ Vertical bars represent SEM. **: $p<0.01$ vs control group

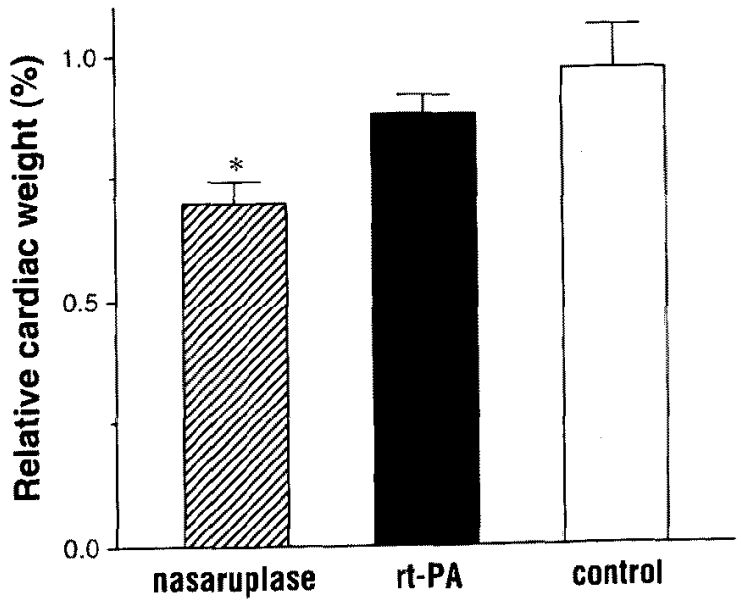

Figure 9. Suppressive effect of nasanplase on left ventricular hypertrophy estimated by relative cardiac weight at sacrifice. Vertical bars represent SEM. *: $p<0.05$ vs control group $\square=$ nasaruplase $(n=7) ; \square=\operatorname{rt}-\mathrm{PA}(n=7), \square=$ control $(n=7)$. 

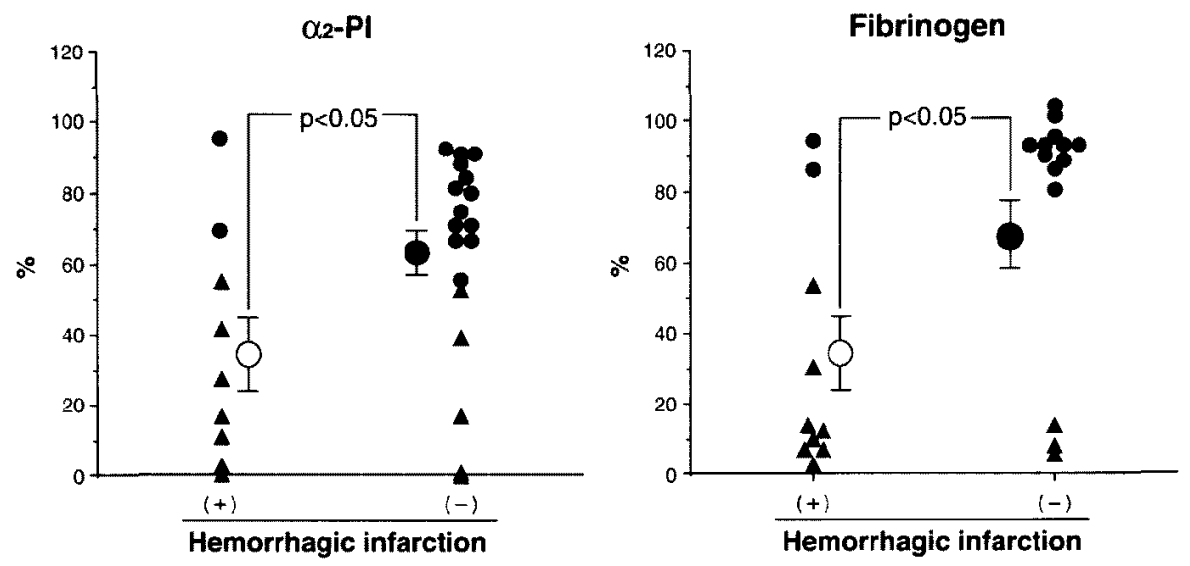

Figure 10. Decrease in plasma concentrations of $\alpha_{2}$-PI and fibrinogen in animals with hemorrhagic infarction.

Plasma concentrations of $\alpha_{2}$-PI and fibrinogen immediately after reperfusion were decreased significantly, compared with those in non-hemorrhagic infarction animals.

$\mathbf{O}=$ nasaruplase; $\mathbf{A}=\mathrm{rt}-\mathrm{PA}$
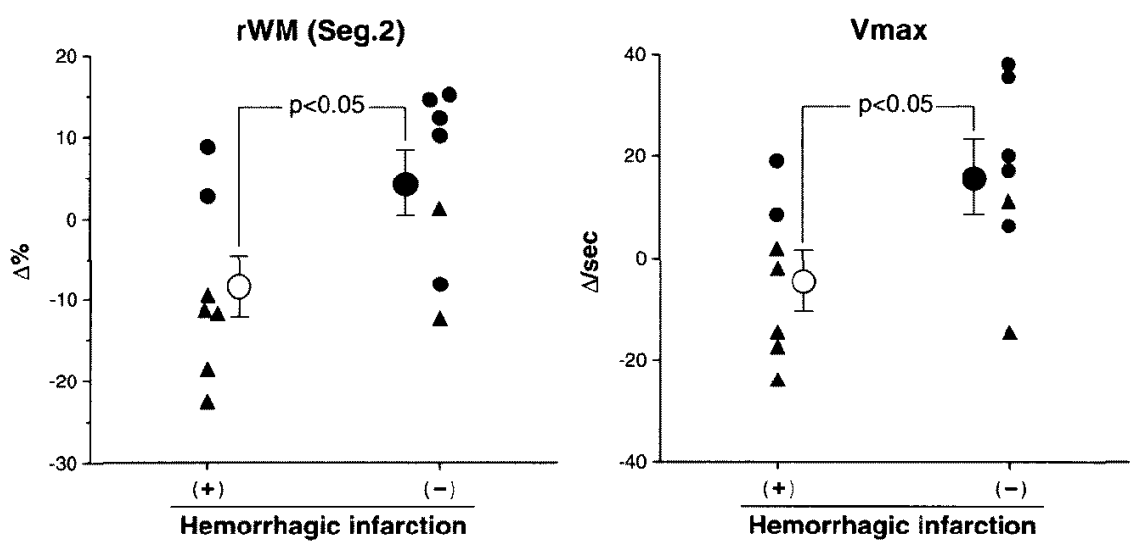

Figure 11. Delay of recovery in left ventricular function in animals with hemorrhagic infarction. Recovery of left ventricular regional wall motion of the anterolateral portion and LV systolic function $\left(V_{\max }\right) 1$ week after reperfusion was statistically inferior to that in non-hemorrhagic infarction animals.

$\boldsymbol{O}=$ nasaruplase; $\boldsymbol{\Lambda}=\mathrm{rt}-\mathrm{PA}$.

nificant decrease in plasma $\alpha_{2}$-PI and fibrinogen concentration occourred immediately after reperfusion in the animals which had hemorrhagic infarction (Figure 10). Moreover, the recovery of left ventricular regional wall motion at the anterolateral portion (Seg. 2) and Vmax after 1 week was inferior in animals with hemorrhagic infarction (Figure 11). Correlation coefficients between the hemorrhagic infarction ratio and the plasma $\alpha_{2}$-PI and fibrinogen concentrations immediately after reperfusion were $-0.740\left(p<0.05 ; \alpha_{2}\right.$-PI $)$ and $-0.798(p<0.05$; 

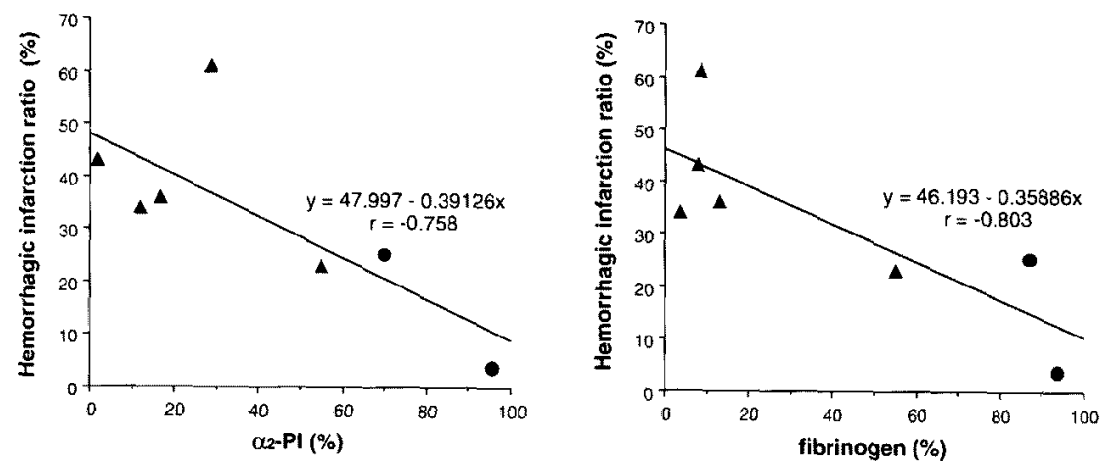

Figure 12. Correlation of hemorrhagic infarction ratio to plasma $\alpha_{2}-\mathrm{PI}$ and fibrinogen concentrations immediately after reperfusion in animals with hemorrhagic infarction. A significant negative correlation was observed between the two parameters. = nasaruplase; $\Delta=$ rt-PA
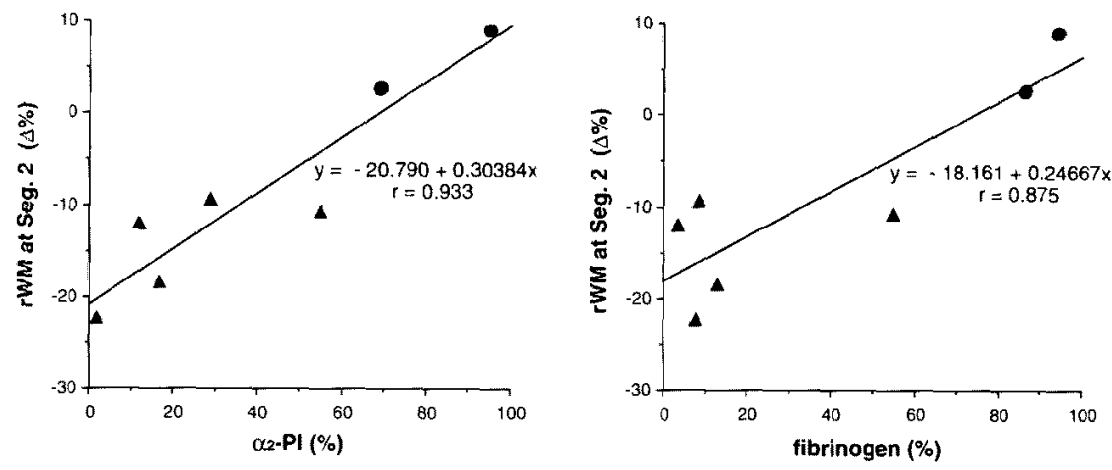

Figure 13. Correlation of recovery of left ventricular regional wall motion at segment 2 after 1 week to plasma $\alpha_{2}$-PI and fibrinogen concentrations immediately after reperfusion in animals with hemorrhagic infarction. The positive correlation between the parameters is significant. $=$ nasaruplase, $\Delta=\mathrm{rt}-\mathrm{PA}$

fibrinogen) (Figure 12). Correlations between these hemostatic changes and the recovery of regional wall motion after 1 week were $0.924\left(p<0.01 ; \alpha_{2}\right.$-PI $)$ or 0.864 ( $p<0.01$; fibrinogen) (Figure 13).

\section{Discussion}

The usefulness of intravenous thrombolytic therapy in the treatment of $\mathrm{AMI}^{19)}$ has motivated the development of new agents having a high affinity for thrombi. Animal models similar to human AMI are indispensable in the development of new drugs. Through intracoronary laser ablation, the present animal 
model provides examples not only of damage to the endothelium but also stenosis of the coronary artery, which are both important factors in the incidence of thrombosis. The stenosis is still observed after 1 week, ${ }^{20)}$ indicating that it is not the result of temporal vasospasm. AMI is induced by selectively inserting a fibrinrich artificial thrombus into the stenosed portion of the coronary artery under closed-chest conditions. Since electrocardiographic observation revealed a transient elevation of ST-segments and T-waves after coronary occlusion that was lowered during subsequent coronary recanalization and reperfusion arrhythmias, the responses in this model resemble AMI in humans. Spontaneous reperfusion did not occur in the control group for up to 1 week. This model was thus considered suitable for the investigation of both acute and chronic effects of thrombolytic agents.

The reperfusion rate of nasaruplase in this model was $78.6 \%$, which is the same as results for both a clinical study $(75.0 \%)^{21)}$ and for recombinant scu-PA (saruplase). ${ }^{22,23)}$ Moreover, no difference in reperfusion rates was observed between nasaruplase and rt-PA $(79.2 \%)$ in the present study. It has been reported that the thrombolytic potency of scu-PA is almost equivalent to that of rt-PA in studies in other animal models. ${ }^{24)}$ Nevertheless, the significant decreases in plasma $\alpha_{2}$-PI and fibrinogen, and the marked increase in PIC were observed only in the rt-PA-treated group immediately after reperfusion. This is due to the fact that nasaruplase is the inactive proenzyme of UK, whereas t-PA itself is active. Accordingly, $\alpha_{2}$-PI concentration was gradually decreased by the addition of rt-PA, but not of nasaruplase, to human plasma in vitro. ${ }^{25)}$ This result suggests that the systemic fibrinolytic action of rt-PA is more potent than that of scu-PA.

It has been suggested that bleeding complications are related to the degree of systemic fibrinolysis. ${ }^{26)}$ Systemic hemorrhage has often been observed during treatment with rt-PA. ${ }^{27)}$ On the other hand, the bleeding complications of scu-PA are benign when compared with those related to rt-PA ${ }^{28)}$ or streptokinase, ${ }^{29)}$ suggesting that scu-PA is a safe thrombolytic agent. ${ }^{23)}$

The frequency of hemorrhagic infarction in the nasaruplase group was significantly lower than in the rt-PA group. The infarct size in the two thrombolytic agent groups was equal, and hemorrhagic infarction did not enlarge the infarct size beyond the reperfused area. However, the ratio of the area of hemorrhagic infarction to total infarct area (hemorrhagic infarction ratio) was higher in the rt-PA group than in the nasaruplase group. In general, the size of hemorrhagic infarction is dependent on reperfusion time, ${ }^{77}$ which was the same in the two thrombolytic agent groups. In the animals with hemorrhagic infarction, a significant negative correlation was found between the hemorrhagic infarction ratio and plasma $\alpha_{2}$-PI and fibrinogen concentrations immediately after reperfusion. From these results, hemorrhagic infarction might be expected to 
extend within the reperfused area, especially in cases with accelerated systemic fibrinogenolysis at the time of rcperfusion.

LVEF and rWM at anterobasal, anterolateral and apical portions worsened as a result of the decrease in cardiac function, including non-infarct areas. These changes reflected both the acute coronary occlusion and the enlargement of infarct area following growth of the thrombus. These parameters recovered the pre-occlusion level after 1 week in the nasaruplase group. The recovery in the rtPA group was poor compared to that in the nasaruplase group. In hemorrhagic infarction animals, rWM was inferior to that in non-hemorrhagic animals. Moreover, a significant positive correlation was found between the recovery of left ventricular function at the area of infarction and systemic fibrinogenolysis. Hemorrhagic infarction delays the recovery of left ventricular function, ${ }^{30,31)}$ duc to microcirculatory damage or an increase in the perfusion pressure of the myocardial tissue. ${ }^{11)}$ The difference between nasaruplase and rt-PA was apparent in Vmax, which is an index of cardiac function at systole. It is thus suggested that massive hemorrhagic infarction may have some effect on left ventricular systolic function.

Increased load on the left ventricle following coronary artery occlusion induces ventricular remodeling ${ }^{32)}$ and reactive hypertrophy ${ }^{33)}$ in the residual myocardium. Nasaruplase suppressed the development of hypertrophy, as a result of early reduction in ventricular loading. rt-PA was less effective than nasaruplase but more effective than the control. Thus, it is suggested that hemorrhagic infarction delays left ventricular remodeling.

All hemostatic parameters returned almost to baseline in all groups after 1 week. There were, however, differences in coronary reocclusion rates between the nasaruplase $(12.5 \%)$ and the rt-PA $(30 \%)$ groups. Perfusion insufficiency of coronary blood flow in the rt-PA group rose to $50 \%$, including high-grade restenosis and circulatory impediment at the apex. In clinical studies, reocclusion rates of rt-PA have been reported to be 5 to $20 \%$ (early phase) up to 24 hours after reperfusion ${ }^{34-367}$ and $30 \%$ (late phase), ${ }^{37)}$ which is higher than after streptokinase therapy. ${ }^{34-36)}$ In addition, mutant t-PA, which has a longer half-life in blood than rt-PA, has shown higher reocclusion rates than rt-PA. ${ }^{38}$ It has been suggested that t-PA has direct or indirect procoagulant effects, such as a rebound increase in plasma coagulation factors. ${ }^{39)}$ In contrast, scu-PA showed a low incidence of both early $(1-1.5 \%)^{40,41)}$ and late $(2.7-5.0 \%)$ reocclusion. ${ }^{21,42)}$ These data suggest that scu-PAs are superior to t-PAs for preventing coronary reocclusion.

\section{ACKNOWLEDGEMENTS}

The authors would like to thank Dr. Y. Uchida and Dr. C. Heldebrant for critical 
comments on the manuscript. The authors also thank Y. Kubo, S. Inoue and Y. Narita for expert technical assistance.

\section{References}

1. Runge MS, Quertermous T, Haber E: Plasminogen activator; the old and the new. Circulation 70: 217,1989

2. Cairns JA, Fuster V, Kennedy JW: Coronary thrombolysis. Chest 102: 4825, 1992

3. Verstraete M, Bernard R, Bory M, Brower RW, Collen D, de Bono DP, Erbel R, Huhmann W, Lennane RJ, Lubsen J, Mathey D, Meyer J, Michel HR, Rutsch W, Schartl M, Schmidt W, Uebis R, von Essen R; Randomised trial of intravenous recombinant tissue-type plasminogen activator versus intravenous streptokinase in acute myocardial infarction. Lancet 1: 842, 1985

4. Neuhaus KL, Tebbe U, Gottwik M, Weber MAJ, Feuerer W, Niederer W, Haerer W, Praetorius F, Grosser KD, Huhmann W, Hoepp HW, Alber G, Sheikzadeh A, Schneider B: Intravenous recombinant tissue plasminogen activator (rt-PA) and urokinase in acute myocardial infarction; results of the German activator urokinase study (GAUS). J Am Coll Cardiol 12: 581, 1988

5. Mueller HS, Rao AK, Forman SA, and the TIMI Investigators: Thrombolysis in myocardial infarction (TIMI); comparative studies of coronary reperfusion and systemic fibrinogenolysis with two forms of recombinant tissue-type plasminogen activator. J Am Coll Cardiol 10: 479, 1987

6. Turi ZG, Goldberg S, LittleJohn JK, VanderArk C, Shadoff N, Karlberg R, Williams J, Butman S, Stadiuus ML, Wise K, Buchbinder M, Genton E, Laskey WK, DeMaria A, White C, Sheehan F, Comp $\mathrm{PC}$, Wynne J, Batson-Fowler G, Edwards S: Dose-related efficacy and bleeding complications of double-chain tissue plasminogen activator in acute myocardial infarction. Am J Cardiol 71: 1009, 1993

7. The TIMI Study Group: Special report; the thrombolysis in myocardial infarction (TIMI) trial. Phase I findings. N Engl J Med 312: 932, 1985

8. Gold HK, Leinbach RC, Garabedian HD, Yasuda T, Johns JA, Grossbard EB, Palacios I, Collen D: Acute coronary reocclusion after thrombolysis with recombinant human tissue-type plasminogen activator; prevention by a maintenance infusion. Circulation 73: 347,1986

9. Marder VJ, Sherry S: Thrombolytic therapy; current status (Part I). N Engl J Med 318: 1512, 1988

10. Marder VJ, Sherry S: Thrombolytic therapy; current status (Part II). N Engl J Med 318: 1585, 1988

11. Sherry S: Appraisal of various thrombolytic agents in the treatment of acute myocardial infarction. Am J Med 83(2A): 31, 1987

12. Gurewich V, Pannell R, Louie S, Kelly P, Suddith RL, Greenlee R: Effective and fibrin-specific clot lysis by a zymogen precursor form of urokinase (pro-urokinase); a study in vitro and in two animal species. J Clin Invest 73: 1731, 1984

13. Kasai S, Arimura H, Nishida M, Suyama T: Proteolytic cleavage of single-chain pro-urokinase induces conformational changes which follows activation of the zymogen and reduction of its high affinity for fibrin. J Biol Chem 260: 12377, 1985

14. Pannell R, Gurewich V: Pro-urokinase; a study of its stability in plasma and a mechanism for its selective fibrinolytic effect. Blood 67: 1215,1986

15. Kasai S, Arimura H, Nishida M, Suyama T: Thrombolytic properties of an inactive proenzyme form of human urokinase secreted from human kidney cells. Cell Struct Funct 10: 151, 1985

16. Kambara H, Kawai C, Kajiwara N, Niitani H, Sasayama S, Kanmatsuse K, Kodama K, Sato H, Nobuyoshi M, Nakashima M, Matsuo O, Matsuda T: Randomized, double-blind multicenter study; comparison of intracoronary single-chain urokinase-type plasminogen activator, pro-urokinase (GE0943), and intracoronary urokinase in patients with acute coronary infarction. Circulation 78: 899, 1988

17. Dodge HT, Sandler H, Baxley WA, Hawley RR: Usefulness and limitation of radiographic methods for determining left ventricular volume. Am J Cardiol 18: 10, 1966

18. Gelberg HJ, Brundage BH, Glamtz S, Parmley WW: Quantitative left ventricular wall motion analysis; a comparison of area, chord and radial methods. Circulation 59: 991, 1979

19. Gruppo Italiano per lo Studio della Streptokinasi nell'Infarto Miocardico (GISSI): Effectiveness of intravenous thrombolytic treatment in acute myocardial infarction. Lancet 1: 397, 1986

20. Weisse AS, Lehan PH, Ettiger PO: The fate of experimentally induced coronary artery thrombosis. 
Am J Cardiol 23: 229, 1969

21. Kordenat RK, Kezdi P, Stanley EL: A new catheter technique for producing experimental coronary thrombosis and selective visualization. Am Heart J 83: 360, 1972

22. Kido $H$, Kubo $\mathrm{X}$, Inoue $\mathrm{S}$, Hayashi $\mathrm{K}$, Narita $\mathrm{Y}$, Uchida $\mathrm{T}$, Watanabe $\mathrm{M}$ : Effects of intravenous administration of nasaruplase, a plasminogen pro-activator, on left ventricular function and systemic hemodynanlics in a canine model of acute myocardial infarction. Folia Pharmacol Japon 101: 79, 1993

23. Kambara H, Kawai C, Nitani H, Kajiwara N, Shinoyama S, Kanmatsuse K, Kodama K, Sato H, Nobuyoshi M, Ishimura T, Kamibatake T, Katori R, Suzuki S, Takahashi M, Takeyama Y, Nakajima H, Mitsufuji K, Yoshida H, Matsuda T, Matsuo S, Nakashima M: A double-blind comparative study between intravenous administration of GE-0943 and urokinase; multicenter study. The Clin Report 27: 889,1993 (in Japanese)

24. Meyer J, Baer F, Barth H, Charbonnier B, El Deeb MF, Erbel R: Randomized double-blind trial of recombinant pro-urokinase against streptokinase in acute myocardial infarction. Lancet 1: 863, 1989

25. Vermeer F, Massberg I, Meyer J, Bar FW, Michels R, Tebbe U: Saruplase, a new fibrin specific thrombolytic agent; efficacy and safety data of 1200 patients. Eur Heart J 12 (Abstr): 29, 1991

26. Hirata $Y$, Umemura $K$, Takiguchi $T$, Uematsu $T$, Nakashima $M$ : A thrombosis model for evaluating thrombolytic agents in the guinea-pig; comparison of t-PA, scu-PA and a novel thrombolytic agent, staphylokinase, on thrombolytic activity. Blood Coagulat Fibrinol 4: 569, 1993

27. Lijinen HR, van Hoef B, de Cock F, Collen D: The mechanism of plasminogen activation and fibrin dissolution by single chain urokinase-type plasminogen activator in plasma milieu in vitro. Blood 73: 1864,1989

28. Rao AK, Pratt C, Berke A, Jaffe A, Ockene I, Schreiber TL: Thrombolysis in myocardial infarction (TIMI) trial-Phase I; hemorrhagic manifestations and changes in plasma fibrinogen and the fibrinolytic system in patients with recombinant tissue plasminogen activator and streptokinase. J Am Coll Cardiol 11: 1,1988

29. Braunwald W, Knatterud GL, Passamani E, Robertson TL, Solomon R: Update from the thrombolysis in myocardial infarction trial (letter). J Am Coll Cardiol 10: 970, 1987

30. Vanhove P: Effects of samplase and of alteplase on hemostasis in patients with acute myocardial infarction, a single-blind, randomized trial. J Am Coll Cardiol 17 (Suppl A): 186A, 1991

31. Erbel R, Windeler J, Meyer J, Mathey D, Bleifeld W, Uebis R: PTCA and thrombolytic treatment with saruplase or streptokinase (PRIMI Study) in acute myocardial infarction. Z Kardiol 80 (Suppl 3): 23,1991

32. Vermeer F, Massberg L, Meyer J, Bar FW, Michels R, Tebbe U: Saruplase, a new fibrin specific thrombolytic agent; efficacy and safety data of 1200 patients. Eur Heart J 12 (Suppl): 29, 1991

33. Fujiwara $H$, Onodera $T$, Tanaka $M$, Fujiwara $T$, Wu DJ, Kawai G, Hamashima Y: A clinicopathologic study of patients with hemorrhagic infarction treated with selective coronary thrombolysis with urokinase. Circulation 73: 749, 1986

34. Mathey DG, Schofer J, Kuch KH, Beil U, Klöpel G: Transluminal, hemorrhagic myocardial infarction after intracoronary streptokinase; clinical, angiographic and necropsy findings. Br Heart J 48: 546,1982

35. Bresnahan GF, Roberts R, Shell WE, Ross JJr, Sobel BE: Deleterious effects due to hemorrhage after myocardial reperfusion. Am J Cardiol 33: 82, 1974

36. Bang NU: Tissue-type plasminogen activator mutants; theoretical and clinical consideration. Circulation 73: 1391, 1989

37. Neuhaus KL, von Essen R, Tebbe U, Vogt A, Roth M, Riess M, Niederer W, Forycki F, Wirtzfeld A, Maeurer W: Cooperative study; improved thrombolysis in acute myocardial infarction with frontloaded administration of alteplase; results of the rt-PA-APSAC patency study (TAPS). J Am Coll Cardiol 19: 885,1992

38. Meijer A, Verheugt FWA, Werter CJPJ, Lie KI, van der Pol JMJ, van Eenige MJ: Aspirin versus coumadin in the prevention of reocclusion and recurrent ischemia after successful thrombolysis; a prospective placebo-controlled angiographic study; results of the APRICOT Study. Circulation 87: 1524,1993

39. Nosaka H, Kimura T, Yokoi H, Hamasaki N, Nobuyoshi M: Comparison with native type t-PA and 
mutant type t-PA in coronary thrombolysis and early reocclusion (in Japanese). Jpn Circ J 57 (Abstr): 591,1993

40. Diefenbach G, Bär F, Meyer J on behalf of the PRIMI Study Group: Early patency and reocclusion rates in acute myocardial infarction; superiority of saruplase (rscu-PA) (SP) to streptokinase (SK). Circulation 82 (Suppl III): 537, 1990

41. Gurewich V: Coronary rethrombosis after successful thrombolysis. Circulation 87: 1759, 1993

42. Diefenbach $\mathrm{C}$ on behalf of the PRIMI Study Group: One-year follow-up in a randomized doubleblind trial of saruplase versus streptokinase in acute myocardial infarction; long-term results of the PRIMI trial. Coronary Artery Dis 3: 925, 1992 